\title{
Cajal Bodies and Oral Squamous Cell Carcinoma
}

\author{
Gargi Sarode $^{1}$, Nikunj Maniyar ${ }^{2}$, Sachin C Sarode ${ }^{3}$, Dharmarajan Gopalkrishnan ${ }^{4}$, Shankargouda Patil ${ }^{5}$
}

The Journal of Contemporary Dental Practice (2019): 10.5005/jp-journals-10024-2573

Oral squamous cell carcinoma (OSCC) is a complex disease with heterogeneity in its clinical and pathological presentation as well as phenotypic and biologic behavior. Despite tremendous advancements in the field of genomics and technology, this particular entity still continues to contribute significantly to the mortality rates worldwide. ${ }^{1}$ Research that is directed toward the identification of molecular strategies adopted by cancer cells for survival, growth, invasion, and migration along with development of novel therapeutic schemes accordingly might help us to overcome this Delphic disease. In recent time, colossal development has been made in the field of cancer research exploring almost all the aspects that are linked with cell physiology. However, entering into totally new territory of cancer biology is the need of the hour that might solve the complex maze of cancer initiation and progression. One such area is Cajal bodies that are yet to be investigated for their role in oral carcinogenesis.

Telomeres are nucleoproteins that cap the ends of chromosomes and guard them from degradation, recombination, and endto-end fusion. Several transcriptional, posttranscriptional, and epigenetic regulations are associated with the maintenance of length of telomere of the chromosomes in cancer cells. A detailed understanding of these mechanisms in-depth may endow us with novel biomarkers and targets for early detection of oral cancers, determining their prognosis, and aid in advancement for effective therapeutics. ${ }^{2}$ Telomerase, a ribonucleoprotein polymerase with a RNA component (hTR) and reverse transcriptase subunit (hTERT), upholds telomere ends by the addition of the telomere reiterate TTAGGG. ${ }^{3}$ The telomerase RNA component (hTR) presents a template for the synthesis of telomere that is catalyzed by telomerase reverse transcriptase (hTERT). ${ }^{2}$ Maintenance of the length of telomere is crucial for genomic solidity and most cancer cells maintain telomeres and divide indefinitely, thus, become immortal through upregulation of hTERT and increased telomerase activity. $^{4}$

Even though the role of telomerase in chromosome maintenance and oncogenesis is well established since years, very little information is available about the crucial steps involved in telomerase synthesis, including the sites for hTR and hTERT localization within the cell nucleus. A work by Narayanan et al. ${ }^{5}$ and Lukowiak et al. ${ }^{6}$ on xenopus oocytes suggests that telomerase RNA localizes to Cajal bodies and nucleoli within the nucleus. Named after Ramon and Cajal ${ }^{7}$ who first described it in neuronal cell nuclei, Cajal bodies or coiled bodies are dynamic, spherical, membraneless structures, measuring about $0.1-2 \mu \mathrm{m}$ in diameter, that are present free within the cell nuclei and/or physically associated to specific regions of chromatin. ${ }^{8}$ The marker protein coilin is almost exclusively used to define the Cajal body in cells using human antibodies and auto-antibodies against it. ${ }^{9}$ Though the exact function of Cajal bodies was very unclear in past, recent studies have found Cajal bodies to be a site for posttranscriptional RNA modification and an assembly of several ribonucleoproteins (RNPs). Results from
${ }^{1-3}$ Department of Oral Pathology and Microbiology, Dr. D. Y. Patil Dental College and Hospital, Dr. D. Y. Patil Vidyapeeth, Pune, Maharashtra, India ${ }^{4}$ Department of Periodontics, Dr. D. Y. Patil Dental College and Hospital, Dr. D. Y. Patil Vidyapeeth, Pune, Maharashtra, India

${ }^{5}$ Department of Maxillofacial Surgery and Diagnostic Sciences, Division of Oral Pathology, College of Dentistry, Jazan University, Kingdom of Saudi Arabia

How to cite this article: Sarode G, Maniyar N, et al. Cajal Bodies and Oral Squamous Cell Carcinoma. J Contemp Dent Pract 2019;20(6):651-652.

Source of support: Nil

Conflict of interest: None

studies based on microinjection discovered that the box H/ACA motif in hTR and other telomerase RNAs play a role in the subcellular localization of the RNA and guide nucleotide modification of snRNAs in the Cajal bodies. It is suggested that the survival motor neuron (SMN) protein and six other proteins of the gemin family facilitates the congregation of hTR and hTERT into active RNPs, including spliceosomes, snoRNPs, and transcriptosomes within Cajal bodies. Moreover, because of its direct interaction with the telomerase-associated protein GAR1, SMN may also play a role in the synthesis of telomerase. ${ }^{9}$

Published data favor the verity that the telomerase activity is easily detected in many malignancies but not in normal cells. Even though hTERT is selectively expressed in cancer cells, hTR is expressed in both normal and cancer cells. Zhu et al. ${ }^{9}$ performed hTR FISH experiment on HeLa cells and found a defined colocalization between hTR and coilin, the protein marker for the Cajal body, highlighting Cajal bodies as the foci for hTR. Yi et al. ${ }^{10}$ previously proposed that the expression of hTR in normal cells is somewhat lower than that expressed in cancer cells. On the contrary, results from the study by Zhu et al. ${ }^{9}$ show that the amount of hTR present in primary somatic cells is statistically comparable to the amount measured for malignant cells suggesting a discrepancy in the hTR distribution among normal and cancer cells; wherein the hTR accumulates within intracellular foci like Cajal bodies in cancer cells that show continuing telomerase activity but not in normal cell lines. Accumulation of hTR in Cajal bodies seems to be a hallmark of different cancer cell types including prostate cancer breast cancer and lung cancer. ${ }^{9}$ The hTR accretion and recognition in nuclear foci including Cajal bodies seem to be reliant on hTERT expression as revealed in the hTERT-transfected fibroblast. Results of FISH by Zhu et al. ${ }^{9}$ also revealed an apparent alliance of hTERT with Cajal bodies in HeLacells, signifying that hTERT is also present in Cajal bodies. It can be, thus, concluded that the Cajal body is the site for interaction between hTR and hTERT. An active enzyme is formed which maintains the length of telomeres in cancer cells making them immortal. Moreover, evidence of accumulation of hTR in Cajal bodies, specifically in cells with ongoing telomerase activity strongly, suggests that the Cajal body is the site of assembly. ${ }^{9}$ 
Thus, this paper will provide a new insight into telomere or telomerase biology in oral cancer cells, while raising several questions about the role of Cajal bodies in oral carcinogenesis. Future studies are needed to resolve these new questions and to identify specific role of Cajal bodies in OSCC pathogenesis.

\section{References}

1. Gupta N, Gupta R, et al. Changing trends in oral cancer-a global scenario. Nepal J Epidemiol. 2016;6(4):613-619.

2. Raghunandan BN, Sanjai K, et al. Expression of human telomerase reverse transcriptase protein in oral epithelial dysplasia and oral squamous cell carcinoma: an immunohistochemical study. J Oral Maxillofac Pathol 2016 Jan-Apr;20(1):96-101.

3. Moyzis RK, Buckingham JM, et al. A highly conserved repetitive DNA sequence, (TTAGGG), present at the telomeres of human chromosomes. Proc Natl Acad Sci USA 1988;85(18):6622-6626.
4. Masutomi K, Hahn WC. Telomerase and tumorigenesis. Cancer Lett 2003;194(2):163-172.

5. Narayanan A, Lukowiak $A$, et al. Nucleolar localization signals of box H/ACA small nucleolar RNAs. EMBO J 1999;18(18):5120-5130.

6. Lukowiak AA, Narayanan A, et al. The snoRNA domain of vertebrate telomerase RNA functions to localize the RNA within the nucleus. RNA 2001 Dec;7(12):1833-1844.

7. Ramon y Cajal S. Un sencillo metodo de coloracionselective del reticuloprotoplasmico y sus efectos en los diversos organos nerviosos de vertebrados e invertebrados. Trab Lab Invest Biol 1903:129-221.

8. Ogg SC, Lamond Al. Cajal bodies and coilin-moving towards function. J Cell Biol 2002 Oct 14;159(1):17-21.

9. Zhu Y, Tomlinson RL, et al. Telomerase RNA accumulates in Cajal bodies in human cancer cells. Mol Biol Cell 2004;15(1):81-90.

10. Yi X, Shay JW, et al. Quantitation of telomerase components and hTERT mRNA splicing patterns in immortal human cells. Nucleic Acids Res 2001;29(23):4818-4825. 